\title{
Search for Dark Matter with the AMANDA and IceCube neutrino detectors $\left(20^{\prime}+5^{\prime}\right)$
}

\author{
Catherine DE CLERCQ* \\ Vrije Universiteit Brussel \\ E-mail: catherine.de.clercq@vub.ac.be
}

\section{for the IceCube collaboration}

If non-baryonic dark matter exists in the form of neutralinos, they may accumulate in massive celestial bodies, leading to a neutrino flux from the decay products of neutralino pair annihilation. Such fluxes can be detected with high energy neutrino telescopes.

Searches were performed for an excess of muon neutrinos produced by neutralino annihilations in the center of the Earth using data collected during approximately 1200 days of detector livetime by the AMANDA detector up to 2003. Searches have also been performed for signals from neutralinos in the Sun using 150 days of data taken with AMANDA in 2003 and 104 days of livetime taken with the 22-string IceCube in 2007. No excess over the expected atmospheric background has been observed. Upper limits at $90 \%$ confidence level have been obtained on the annihilation rates of captured neutralinos and the resulting muon flux, as function of the neutralino mass in the range $50-5000 \mathrm{GeV}$.

We also discuss the improvements expected from the full IceCube detector and from the combination with the planned DeepCore low energy extension.

Identification of dark matter 2008

August 18-22, 2008

Stockholm, Sweden

\footnotetext{
${ }^{*}$ Speaker.
} 


\section{Introduction}

Cosmological observations suggest the presence of dark matter on all distance scales. One of the candidates for cold dark matter is the lightest neutralino, $\tilde{\chi}_{1}^{0}$, of the MSSM with R-parity conservation. The mass of this particle is expected to be in the $\mathrm{GeV}-\mathrm{TeV}$ range [1]. On their trajectory through the Universe the neutralinos will scatter on normal matter and lose energy. Eventually, they will be trapped in the gravitational field of heavy celestial objects, like the Earth and the Sun [2], where they can annihilate pairwise in Standard Model particles. The neutrinos produced in the decays of these particles can then be detected as an excess over the expected atmospheric neutrino flux. In this paper results are presented of searches with the AMANDA and IceCube detectors for signals of neutralino dark matter accumulated in the Earth and the Sun.

The AMANDA detector [3] at the South Pole is an array of optical modules which detect Cherenkov light from charged particles that are produced in neutrino interactions in the polar ice cap. It consists of 677 optical modules distributed on 19 vertical strings (AMANDA II). Before 2000, the detector consisted of between 10 and 13 strings (AMANDA B10). IceCube [4] is an array of about one cubic km, which will consist of 4800 digital optical modules (DOM) spread over 80 strings. In 200722 strings were operational (IceCube-22 detector); the full detector will be completed in 2011.

The dark matter searches are based on the detection of muons from $v_{\mu}$ interactions. For a detector at the South Pole, muons from a neutralino-induced signal will yield vertical upwardgoing (Earth) or nearly horizontal (Sun) tracks. The selection of neutralino induced muons is performed in a series of filtering steps. The first levels of filtering aim at reducing the atmospheric muon background by a factor of about $10^{6}$. After these cuts, the data consist mainly of muons from atmospheric $v_{\mu}$ and a possible signal. The filters were generally tuned separately for each MSSM model studied. The optimisation of the filters was done with events simulated with WIMPSIM [5]. Seven neutralino masses between $50 \mathrm{GeV}$ and $5000 \mathrm{GeV}$ were considered, and two annihilation channels for each mass: the $\tilde{\chi}_{1}^{0} \tilde{\chi}_{1}^{0} \rightarrow W^{+} W^{-}$channel produces a hard neutrino energy spectrum ( $\tilde{\chi}_{1}^{0} \tilde{\chi}_{1}^{0} \rightarrow \tau^{+} \tau^{-}$for a $50 \mathrm{GeV}$ neutralino), while the $\tilde{\chi}_{1}^{0} \tilde{\chi}_{1}^{0} \rightarrow b \bar{b}$ channel yields a soft spectrum. The hadronic physics and $\mathrm{W}$ decays were modelled with PYTHIA [6], with a correction to account for $\mathrm{b}$ meson interactions before decay. For the background studies, the showers which give rise to the atmospheric muon background were generated with CORSIKA [7] while the atmospheric upward-going neutrinos were simulated according to the spectrum from ref. [8].

\section{Search for a signal of neutralino annihilation in the center of the Earth}

A search for neutralino-induced muons from the center of the Earth was performed with AMANDA B10 data collected between 1997 and 1999, corresponding to a detector livetime of 536 days. The details of this analysis can be found in ref. [9]. No evidence for a signal was found and an upper limit was set on the neutrino-to-muon conversion rate, which can be related to an upper limit on the muon flux (using DARKSUSY [10]). The results for neutralino masses above $250 \mathrm{GeV}$ and for the hard annihilation channels are shown in Fig. 1. In a more recent analysis [11], the AMANDA II data taken in 2001-03 (689 days livetime) were searched for a similar signal with emphasis on the low neutralino masses $\left(\mathrm{m}_{\tilde{\chi}} \leq 250 \mathrm{GeV}\right)$. A large improvement in sensitivity 

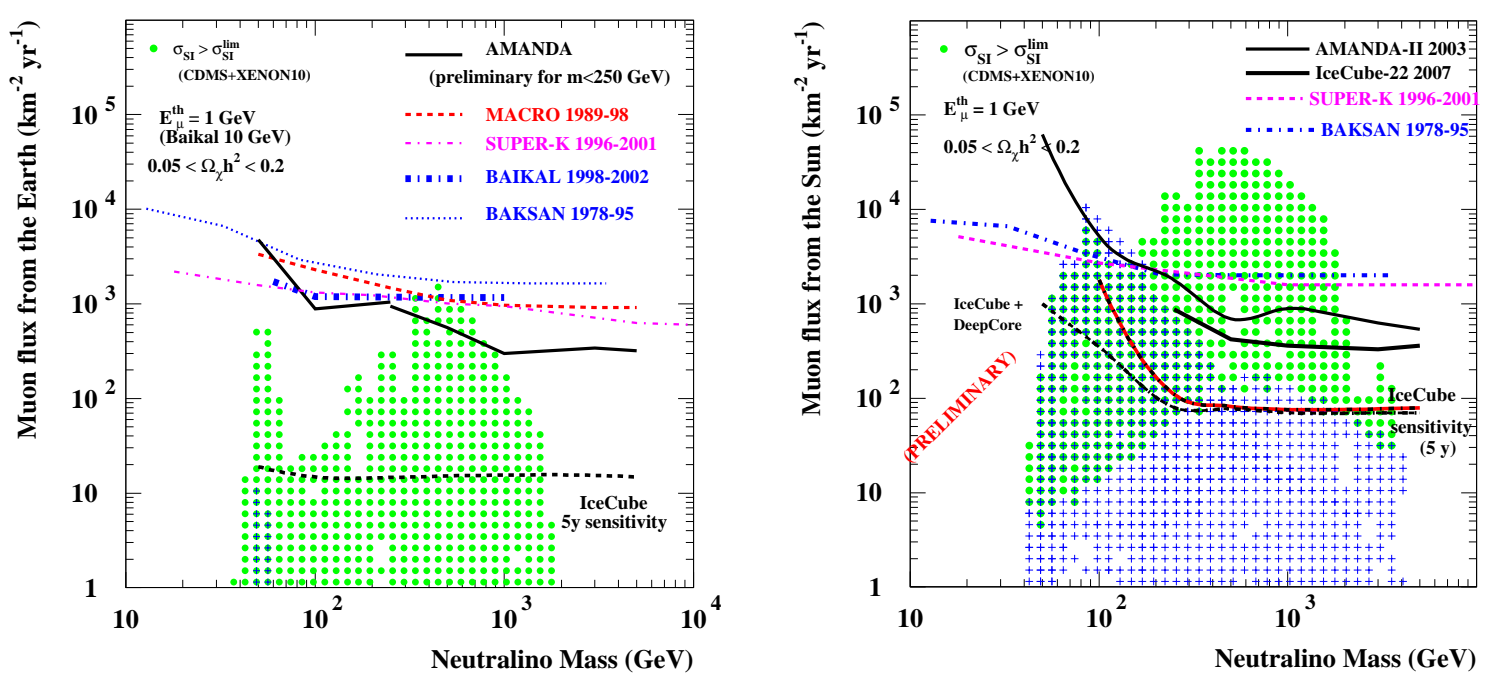

Figure 1: $90 \%$ confidence level upper limits on the muon flux from hard neutralino annihilations in the center of the Earth (left) and in the Sun (right), compared to other indirect and to direct searches, see text.

was obtained thanks to a dedicated low energy trigger. No evidence for a signal was found; the upper limits on hypothetical muon fluxes from neutralino annihilation are also shown in figure 1. All results shown include systematic errors which amount to around $30 \%$. The main sources of systematic errors are the parametrization of the ice properties, the modelling of the optical module sensitivity and the calculation of the expected atmospheric neutrino flux.

\section{Search for a signal from neutralino annihilation in the Sun}

A first attempt to use the AMANDA II data in a search for muons from solar neutralino annihilation showed that the reconstruction of near horizontal tracks was of sufficiently good quality to obtain an angular resolution of 3-5 degrees, depending on the $v_{\mu}$ energy [12]. Improved sensitivities have been obtained since by a better understanding of the detector (ice properties) and a dedicated low energy trigger. The AMANDA II data taken in 2003 (150 days livetime with Sun below horizon) were searched for a signal from solar neutralinos based on these improvements [13]. Similarly as for the neutralino search explained in section 2, the filtering of the data was done in several steps and optimised for each model separately. To evaluate the signal content in the final event sample, hypothesis testing was done based on $\Psi$, the angle between the reconstructed track and the direction of the Sun. The signal and background probability density functions were taken from simulation and from off-source data respectively.

The IceCube data taken in 2007 (104 days livetime with Sun below horizon) were also searched for muons from neutralino annihilations in the Sun [14]. Because of the sparser spacing of the DOMs, this analysis was focussed on neutralino masses of $250 \mathrm{GeV}$ and higher. The analysis strategy was similar to what was done with the 2003 AMANDA data (see above). In both searches there was no evidence for a signal. A $90 \%$ confidence level upper limit was set on the neutrino-to-muon 
conversion rate, and the related muon flux (see figure 1). The flux limits shown for the IceCube-22 analysis include systematic errors, which amount to 19-25\% depending on the neutralino mass. This is the first measurement of limits with IceCube and amongst of the most stringent on neutralino annihilations in the Sun.

\section{Discussion and outlook}

Figure 1 shows the AMANDA and IceCube upper limits on the muon flux from neutralino annihilations into hard neutrinos in the Earth and the Sun, together with the results from other indirect searches [15]. The limits have been rescaled to a common muon energy threshold of $1 \mathrm{GeV}$ using DARKSUSY [10]. Also shown are the cosmologically relevant MSSM models [2] allowed (markers) and disfavoured (dots) by the direct search results from CDMS [16] and XENON10 [17]. We are currently performing searches for a dark matter signal from the Sun with the full 2001-06 AMANDA data set. The increased detector exposure combined with the new trigger setting will result in a considerable improvement in the sensitivity.

The IceCube detector, to be completed by 2011, will encompass an approximately hexagonal instrumented volume of $1 \mathrm{~km}^{3}$. After 5 years of data taking in its final configuration, IceCube will be sensitive to fluxes from neutralino annihilations in the Earth which are of the order of 20 muons per $\mathrm{km}^{2}$ per year. Similarly, with 5 years of data taken with the Sun below the horizon, IceCube will be sensitive to fluxes of the order of 100 muons per $\mathrm{km}^{2}$ per year from neutralino annihilations in the Sun (see fig. 1).

The IceCube collaboration plans to extend the detector with Deep Core, a dense core of 360 DOMs placed in deep very clear ice [18]. This low energy extension will benefit from the surrounding IceCube strings as atmospheric veto, and will allow to extend the sensitivity for dark matter searches down to $50 \mathrm{GeV}$ (see fig. 1). Moreover, this detector will have a $4 \pi$ sensitivity for neutrinos in the GeV-TeV energy range, and open up the field of view to the Southern hemisphere and the galactic center.

\section{References}

[1] G. Jungman, M. Kamionkowski and K. Griest, Phys. Rept. 267, 195 (1996).

[2] W.H. Press and D.N. Spergel, Astrophys. J. 296, 679 (1985); J. Lundberg and J. Edsjö, Phys. Rev. D69, 123505 (2004).

[3] J. Ahrens et al. (the AMANDA Collaboration), Nucl. Instr. Meth. A524, 169 (2004).

[4] A. Achterberg et al. (the IceCube Collaboration), Astrop. Phys. 26, 155 (2006).

[5] M. Blennow et al., Journ. of Cosm. \& Astrop. Phys. 01, 021 (2008).

[6] T. Sjöstrand, S. Mrenna and P. Skands, PYTHIA 6.4 Physics and Manual, J. High Energy Phys. 05, 026 (2006).

[7] D. Heck et al., FZKA report 6019 (1998).

[8] A. Gazizov and M. Kowalski, astro-ph/0406439 (2004); G.D. Barr et al., Phys. Rev. D70, 023006 (2004).

[9] A. Achterberg et al. (the IceCube Collaboration), Astropart. Phys. 26,129 (2006).

[10] P. Gondolo et al., Journ. of Cosm. \& Astrop. Phys. 0407, 008 (2004). 
[11] Anna Davour, Ph.D. thesis, Uppsala University, 2007.

[12] M. Ackermann et al. (the AMANDA Collaboration), Astropart. Phys. 24, 459 (2006).

[13] Thomas Burgess, Ph.D. thesis, Stockholm University, 2008.

[14] The IceCube collaboration, in preparation.

[15] M. Boliev et al. (the BAKSAN Collaboration), in Proc. of Dark Matter in Astro- and Particle Physics, edited by H.V. Klapdor-Kleingrothaus and Y. Ramachers (World Scientific, 1997); M. Ambrosio et al. (the MACRO Collaboration), Phys. Rev. D60, 082002 (1999); S. Desai et al. (the Super-Kamiokande Collaboration), Phys. Rev. D70, 083523 (2004), erratum ibid D70, 109901 (2004); V. Aynutdinov et al. (the Baikal Collaboration), Nucl Phys B (Proc. Suppl.) 143, 335 (2005).

[16] Z. Ahmed et al. (the CDMS collaboration), arXiv:0802.3530v2 [astro-ph].

[17] J. Angle et al., (the XENON collaboration), Phys. Rev. Lett. 100, 021303 (2008).

[18] E. Resconi, Status and prospects of the IceCube neutrino telescope, arXiv 0807.3891v1 [astro-ph]. 